\title{
Potentiation of Gamma Oscillatory Activity through Repetitive Transcranial Magnetic Stimulation of the Dorsolateral Prefrontal Cortex
}

\author{
Mera S Barr', Faranak Farzan', Pablo M Rusjan², Robert Chen ${ }^{3}$, Paul B Fitzgerald ${ }^{4}$ and Zafiris J Daskalakis*,', \\ 'Schizophrenia Program, Centre for Addiction and Mental Health, Department of Psychiatry, University of Toronto, Toronto, Ontario, Canada; \\ ${ }^{2}$ PET Centre, Centre for Addiction and Mental Health, Toronto, Ontario, Canada; ${ }^{3}$ Division of Neurology, Toronto Research Institute, University of \\ Toronto, Toronto, Ontario, Canada; ${ }^{4}$ Department of Psychological Medicine, Alfred Psychiatry Research Centre, The Alfred and Monash University, \\ Melbourne, Victoria, Australia
}

\begin{abstract}
Neuronal oscillations in the gamma $(\gamma)$ frequency range $(30-50 \mathrm{~Hz})$ have been associated with cognition. Working memory (WM), a cognitive task involving the on-line maintenance and manipulation of information, elicits increases in $\gamma$ oscillations with greater cognitive demand, particularly in the dorsolateral prefrontal cortex (DLPFC). The generation and modulation of $\gamma$ oscillations have been attributed to inhibitory interneuron networks that use $\gamma$-aminobutyric acid (GABA) as their principal neurotransmitter. Repetitive transcranial magnetic stimulation (rTMS) represents a non-invasive method to stimulate the cortex that has been shown to modify cognition and GABA inhibitory mechanisms, particularly with higher frequencies (ie, 10-20 Hz). We measured the effect of high-frequency rTMS applied to the DLPFC on $\gamma$-oscillations elicited during the N-back WM task in healthy individuals. Active rTMS significantly increased $\gamma$-oscillations generated during the $N$-back conditions with the greatest cognitive demand. Further, no significant changes were found in other frequency ranges, suggesting that rTMS selectively modulates $\gamma$-oscillations in the frontal brain regions. These findings provide important insights into the neurophysiological mechanisms that underlie higher-order cognitive processes, and suggest that rTMS may be used as a cognitive enhancing strategy in neuropsychiatric disorders that suffer from cognitive deficits.
\end{abstract}

Neuropsychopharmacology (2009) 34, 2359-2367; doi:I0. I038/npp.2009.79; published online I5 July 2009

Keywords: cognition; DLPFC; EEG; gamma oscillations; rTMS; working memory

\section{INTRODUCTION}

The importance of gamma $(\gamma)$ oscillatory activity in higher cognitive tasks is an area of great interest and has recently been shown as a key neurophysiological mechanism underlying working memory (WM). Commonly defined as the ability to maintain and manipulate information over short periods of time (Baddeley, 1986), WM is often argued as the essence of all prefrontal functions because of its importance in everyday complex cognitive tasks, such as language comprehension, learning, and reasoning (Baddeley, 1992, 2000). Moreover, the dorsolateral prefrontal cortex (DLPFC) is consistently reported to mediate WM processes, which are revealed through enhanced blood oxygen level-dependent (BOLD) activity in functional

\footnotetext{
*Correspondence: Dr ZJ Daskalakis, Schizophrenia Program, Centre for Addiction and Mental Health, Department of Psychiatry, University of Toronto, 7th Floor-Clarke Division, 250 College Street, Toronto, Ontario, Canada, Tel: (4I6) 535-850I ext. 4319, Fax: (4I6) 979-6936, E-mail: Jeff_Daskalakis@camh.net
}

Received 25 March 2009; revised 29 May 2009; accepted I June 2009 magnetic resonance imaging (fMRI) studies (Petrides, 2000; Owen, 1997).

Several cognitive paradigms are used to index WM. For example, the $N$-back task requires participants to determine whether the current stimulus is the same stimulus that was presented ' $N$ ' trials back, thus allowing the evaluation of increasing cognitive demand (ie, WM load) on $\gamma$-oscillatory activity. Further, a growing body of evidence has shown increased $\gamma$-oscillatory activity with cognitive demand (Howard et al, 2003; Meltzer et al, 2008; Basar-Eroglu et al, 2007). For example, Cho et al (2006) reported that $\gamma$-oscillatory activity increased with the increased cognitive control in the frontal regions that was related to performance (Cho et al, 2006), suggesting that $\gamma$ is modulated by cognitive demand, which may underlie WM performance. It has been proposed that $\gamma$-aminobutyric acid (GABA) inhibitory interneurons in the DLPFC contribute to the generation and synchronization of pyramidal neurons necessary for optimal WM performance (Wang and Buzsaki, 1996; Traub et al, 2004). For instance, Wilson et al (1994) reported fast-spiking GABAergic neuron activity in the DLPFC during the delay period of WM tasks, 
whereas the injection of GABA antagonist bicuculline disrupts WM performance in monkeys (Sawaguchi et al, 1989).

Repetitive transcranial magnetic stimulation (rTMS) delivers repeated magnetic pulses to the cortex to induce plasticity-like changes in cortical function and behavior. For example, high-frequency rTMS has been shown to improve language functions in healthy individuals (Sparing et al, 2001), as well as to improve different aspects of memory in major depressive disorder patients (Little et al, 2000; Martis et al, 2003; O'Connor et al, 2005). In addition, rTMS on the motor cortex has been shown to enhance GABA-mediated inhibitory neurotransmission in healthy individuals - that is, Daskalakis et al (2006) reported a lengthening of the cortical silent period (CSP), a measure reflective of $\mathrm{GABA}_{\mathrm{B}}$ mediated inhibitory neurotransmission (Ziemann et al, 1996) with increased stimulation frequency, which was maximal at $20 \mathrm{~Hz}$ (Daskalakis et al, 2006). High-frequency (ie, $20 \mathrm{~Hz}$ ) rTMS thus represents a possible mechanism through which to potentiate $\gamma$-oscillatory activity, which may, in turn, improve WM performance.

This study, therefore, aimed to evaluate the effect of $20 \mathrm{~Hz}$ rTMS applied to the right and left DLPFC on $\gamma$-oscillatory activity elicited during the $\mathrm{N}$-back task in healthy individuals. We hypothesized that active rTMS would enhance $\gamma$-oscillatory activity with increased WM load with no effect on the oscillatory activity in other frequency ranges (ie, $\delta, \theta$, $\alpha$, and $\beta$ ).

\section{MATERIALS AND METHODS}

\section{Participants}

A total of 22 right-handed healthy volunteers participated in this study (mean age $=34.2$ years, $\mathrm{SD}=7.16$ years, range $=24-49$ years; 11 men and 11 woman). Handedness was confirmed using the Oldfield Handedness Inventory (Oldfield, 1971). All participants gave their written informed consent and the protocol was approved by the Centre for Addiction and Mental Health in accordance with the Declaration of Helsinki. Exclusion criteria included a selfreported comorbid medical illness or a history of drug or alcohol abuse. Moreover, psychopathology was ruled out through the personality assessment screener (PAS; Psychological Assessment Resources).

\section{Procedure}

Before the experiment, participants were randomized into two groups allocated to receive either active or sham rTMS. Participants were blinded to their group assignment until the completion of the study to avoid individual biases. The experiment took place over 2 testing days. On the first day, participants performed the $N$-back test while their EEG activity was being recorded. One week later, rTMS was administered over the DLPFC before the final testing in the $N$-back task. The final $N$-back task was performed $\sim 20 \mathrm{~min}$ after rTMS administration to allow for cortical plasticity changes to take place as well as for the placement of the EEG cap. These two $N$-back testing sessions will hereafter be referred to as 'pre' and 'post' measures relative to rTMS administration.

\section{$N$-Back Task}

Participants performed the $N$-back task while their EEG activity was being recorded (STIM2, Neuroscan) before (pre) and after (post) a single session of rTMS to the DLPFC. During this task, stimuli were presented on a computer monitor one at a time to the participants who were required to push one button (target) if the present stimulus was identical to the stimulus presented ' $N$ ' trials back; otherwise, the participants pushed a different button (non-target). Thus, the effect of increasing the cognitive demand on $\gamma$-oscillatory activity was tested by varying the ' $N$ ' of the task in the $0-, 1-, 2-$, and 3-back conditions (Figure 1a). It is noted that in the 0-back condition, participants were required to push the non-target button every time a stimulus was presented, and thus did not have a memory component. Stimuli consisted of black capital letters presented for $250 \mathrm{~ms}$ followed by a delay period of $3000 \mathrm{~ms}$ during which the participant was required to respond before the presentation of a plus sign for $1305 \mathrm{~ms}$ indicating the end of this trial (Figure 1b). In the 0-, 1-, and 2-back condition, stimuli were presented continuously for $15 \mathrm{~min}$, whereas in the 3-back condition stimuli were presented for $30 \mathrm{~min}$. The 3 -back condition was administered for double the length of the other conditions $(30 \mathrm{~min}$ rather than $15 \mathrm{~min}$ ) to ensure that the frequency of target letters in this condition was comparable with those presented in the 1and 2-back conditions, as these target letters occurred less frequently (ie, at least 3 letters back). Thus, the proportion of target letters in each of the conditions was 23.1, 15.8, and $14.8 \%$ in the 1-, 2-, and 3-back conditions, respectively. Consequently, this also ensured that a satisfactory number of correct responses were contained in all of the conditions for the data analysis (Table 1). The total time for participants to complete the $N$-back task was $1 \mathrm{~h}$ and $15 \mathrm{~min}$ with the presentation of the conditions randomized and counterbalanced to prevent order effects.

\section{Repetitive TMS}

Repetitive TMS was administered using a Medtronic MagPro stimulator (Medtronic) with a $70-\mathrm{mm}$ diameter figure-of- 8 coil to the right and left DLPFC at $20 \mathrm{~Hz}$,

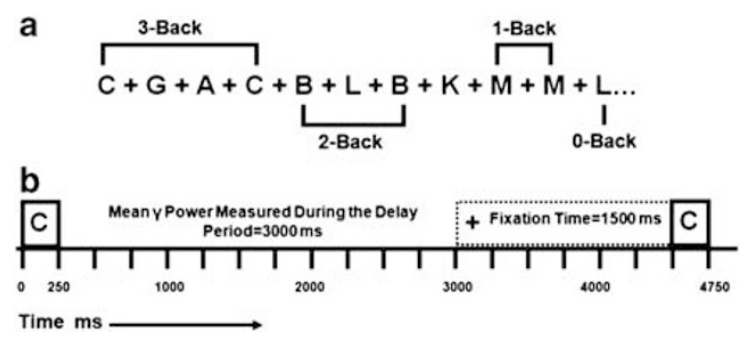

Figure I N-back WM task. (a) A representation of the four different WM conditions ( $0,1,2$, and 3 -back) that were administered in a randomized order to participants before and after rTMS. (b) The timing of one trial from the presentation of a one letter separated by a $(+)$ sign followed by a subsequent letter for a total time of $3000 \mathrm{~ms}$. Participants were required to push one button (target) if the present letter was identical to the letter presented ' $N$ ' trials back; otherwise, the participants pushed a different button (non-target). 
Table I Mean Number of Trials (TC+NTC) Following Artifact Correction for each $\mathrm{N}$-back Condition Pre- and Post rTMS Administration

\begin{tabular}{|c|c|c|c|c|c|c|c|c|}
\hline \multirow[t]{2}{*}{ Group } & \multicolumn{4}{|c|}{ Pre rTMS } & \multicolumn{4}{|c|}{ Post rTMS } \\
\hline & 0-back & I-back & 2-back & 3-back & 0-back & I-back & 2-back & 3-back \\
\hline Sham & $14 \mid .7$ & 127.9 & | 49.7 & 283.9 & 125.9 & 134.3 & 139.8 & 264.5 \\
\hline Active & 127.9 & 137.6 & 124.4 & 225.8 & 103.6 & 129.4 & 111.7 & 185.6 \\
\hline
\end{tabular}

$90 \%$ resting motor threshold for 25 trains comprising 30 pulses per train, inter-train interval of $30 \mathrm{~s}$ for a total of 750 pulses per hemisphere and in accordance with published safety guidelines (Chen et al, 1997). The time of the rTMS delivery was $25 \mathrm{~min}, 12.5 \mathrm{~min}$ per hemisphere. The resting motor threshold was defined as the lowest intensity that produced a motor-evoked potential of at least $50 \mu \mathrm{V}$ in $50 \%$ of the trials delivered. Sham stimulation was delivered with the same rTMS parameters as active stimulation with the coil held in a single wing-tilt position at $90^{\circ}$ to induce similar somatic sensations as in the active stimulation with minimal direct brain effects. The order of stimulation (right, then left $v s$ left, then right) was also randomized and counterbalanced to prevent order effects.

\section{DLPFC Site Localization}

The localization of the DLPFC was determined through neuronavigational techniques using the MINIBIRD system (Ascension Technologies) combined with MRIcro/reg software using a T1-weighted MRI scan obtained for each participant with seven fiducial markers in place. Repetitive TMS was targeted at the junction of the middle and anterior one-third of the middle frontal gyrus (Talairach coordinates $(x, y, z)=-50,30,36)$, corresponding with the posterior regions of the Brodmann area 9 (BA9), and overlapping with the superior region of BA46 (Figure 2). The selection of this site was based on a recent meta-analysis of functional imaging studies that examined WM and the activation of the DLPFC (Cannon et al, 2005; Mendrek et al, 2005; Tan et al, 2005).

\section{EEG Measurement of Evoked $\boldsymbol{\gamma}$-Oscillatory Activity}

Evoked oscillatory responses are phase locked to the stimulus onset with a fixed latency within the first $100 \mathrm{~ms}$ after stimulus onset and, therefore, can be measured by stimulus-triggered averaging of responses (Tallon-Baudry et al, 1999). In contrast, induced oscillatory activity is not phase locked to stimulus onset and appears as a jitter in latency that varies from trial to trial; thus, these responses are canceled out when trials are averaged (Tallon-Baudry et al, 1999). Both evoked and induced $\gamma$-activities have been associated with sensory and cognitive processing (Lutzenberger et al, 1995; Muller et al, 1996; Cho et al, 2006). Moreover, induced $\gamma$-oscillatory activity has been shown to increase with increased cognitive control (Cho et al, 2006), whereas other studies have shown that evoked $\gamma$-oscillatory activity increases with cognitive demand in

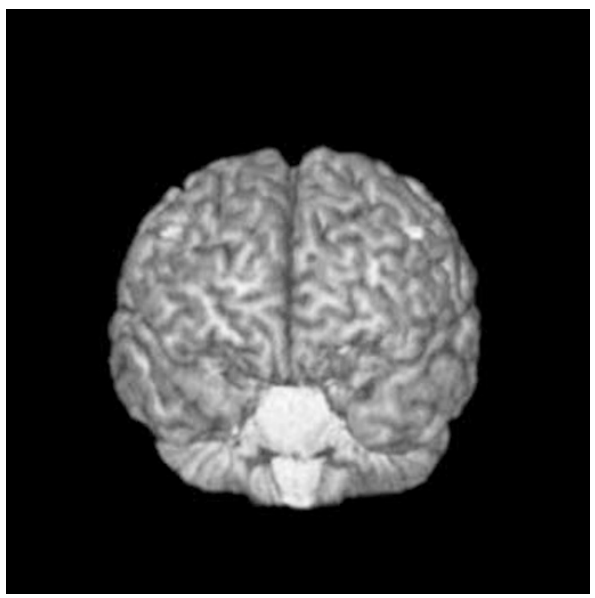

Figure 2 Targeting the dorsolateral prefrontal cortex to index cortical inhibition. Transverse view from a single participant with exposed cortex and overlap of Brodmann areas 9 and 46, highlighted (white) on a TIweighted 3D MRI. Using MRI-to-MiniBird co-registration, the center of the TMS coil was held over this region.

WM paradigms (Howard et al, 2003; Basar-Eroglu et al, 2007; Meltzer et al, 2008). As such, we measured the mean evoked $\gamma$-power from frontal electrodes while the participants completed the $N$-back task before (pre) and after (post) rTMS was administered over the right and left DLPFC.

\section{EEG Recording}

EEG data were acquired using a 64-electrode cap and Synamps2 DC-coupled EEG system (Compumedics). Four electrodes placed on the outer side of each eye, above, and below the left eye were used to monitor the eye movement artifact. Data were recorded at a rate of $1000 \mathrm{~Hz}$ DC and with a $0.3-200 \mathrm{~Hz}$ band pass hardware filter. Electrode impedances were lowered to $<5 \mathrm{k} \Omega$. All channels were referenced to an electrode placed posterior to the $\mathrm{Cz}$ electrode.

\section{Offline EEG Processing}

To measure the mean evoked $\gamma$-power, data were filtered off-line using a $1-100 \mathrm{~Hz}$ band pass zero-phase-shift filter (slope, $24 \mathrm{~dB} / \mathrm{oct}$ ). Epochs were defined as -1000 to $+3095 \mathrm{~ms}$ relative to the cue onset and were baseline corrected with respect to the prestimulus interval $(-1000$ to cue onset). To measure the evoked $\gamma$-power, we selected the entire delay period of $3000 \mathrm{~ms}$ relative to the stimulus because the $N$-back task requires the continuous maintenance of ' $N$ ' letters in preparation for the next trial as the task runs sequentially for $\sim 15-30$ min depending on the task condition. All trials were manually inspected and any error trials or epochs containing artifact (movement or electrooculogram exceeding $\pm 50 \mu \mathrm{V}$ ) were excluded from further analysis.

\section{Data Analysis}

Artifact-free EEG data were imported into MATLAB (The MathWorks, Natick, MA) using the EEGLAB toolbox (Delorme and Makeig, 2004) for subsequent analysis. Evoked 
$\gamma$-power in the $30-50 \mathrm{~Hz}$ range was averaged over the delay period (0-3000 ms from cue onset) for the target correct (TC) and non-target correct (NTC) responses for each WM load pre- and post rTMS for each participant. The mean evoked $\gamma$ power was then assessed during these responses $(\mathrm{TC}+\mathrm{NTC})$ in the frontal electrodes (AF3, AF4, F5, F3, F1, FZ, F2, F4, and F6), and averaged for each individual. As the spectral analysis of EEG activity is often not normally distributed (Bender et al, 1992), the data were log-transformed before analysis. Full-factorial mixed model repeated measures (MMRM) analysis was performed with rTMS (active $v s$ sham) as a between-subject factor with time (pre $v s$ post) and WM load (0- vs 1-vs 2- vs 3-back) as within-subject factors on the data with a significance level set at $p<0.05$. Bonferroni-adjusted pairwise comparisons were then performed (SAS System v.9.1.3; SAS Institute, Cary, NC, USA).

\section{RESULTS}

\section{Evoked $\gamma$-Power}

As expected, the MMRM analysis revealed an effect of WM load on mean evoked $\gamma$-power $\left(F_{(3,60)}=18.59, p<0.0001\right)$, with participants generating the greatest $\gamma$-power during the 2-back condition compared with the $0-(p<0.0001)$, 1 - $(p=0.0416)$, and 3-back $(p=0.0004)$ conditions, respectively (Figure 3a). Furthermore, the effect of time on $\gamma$-power was also found to be significant $\left(F_{(1,20)}=29.49\right.$, $p<0.0001)$ with greater $\gamma$-power elicited during the $N$-back after rTMS stimulation (post) compared with baseline (pre). Finally, the effect of rTMS on $\gamma$-power was also significant $\left(F_{(1,20)}=9.26, p=0.0064\right)$, with participants in the active group generating higher $\gamma$-power during the $N$-back task compared with those who received sham stimulation.

The MMRM analysis on mean $\gamma$-power also revealed significant interaction effects (Figure 3a). In particular, an interaction was found between time and rTMS $\left(F_{(1,20)}=12.43, p=0.0021\right)$ with active stimulation resulting in greater $\gamma$-power compared with baseline (pre; $p<0.0001$ ) and with sham rTMS (post; $p=0.0028$ ). There was also a significant time $\times$ WM load $\times$ rTMS interaction: $\left(F_{(3,60)}=3.79, p=0.0148\right)$. Importantly, although there was no difference in $\gamma$-power during the $N$-back task at baseline (pre) between the active and sham groups, active rTMS resulted in a significant increase in $\gamma$-power in the 2$(p=0.0032)$ and 3-back $(p=0.0288)$ conditions compared with the sham group. Altogether, these findings suggest that active rTMS resulted in a significant potentiation in $\gamma$-power that was greatest in the $\mathrm{N}$-back conditions with the greatest WM load. Figure 4a shows the mean absolute change in $\gamma$ power as topographical illustrations (represented by hot colors i.e., red $=$ maximal, blue $=$ minimal values). Inspection of the topographical plots revealed a maximal change in $\gamma$-power in the frontal brain regions compared with other cortical regions after active stimulation. As such, a repeated-measures ANOVA was performed comparing the mean sum change in $\gamma$-power in five frontal electrodes (FPZ, FP1, FP2, AF3, and AF4) selected on the basis of hot-colored electrodes from the topographical plot with that in five electrodes from the posterior region $(\mathrm{OZ}, \mathrm{O} 1, \mathrm{O} 2, \mathrm{PO}$, and PO4), with rTMS as a between-subject factor. A significant effect of the brain region was found $\left(F_{(1,19)}=14.938\right.$,

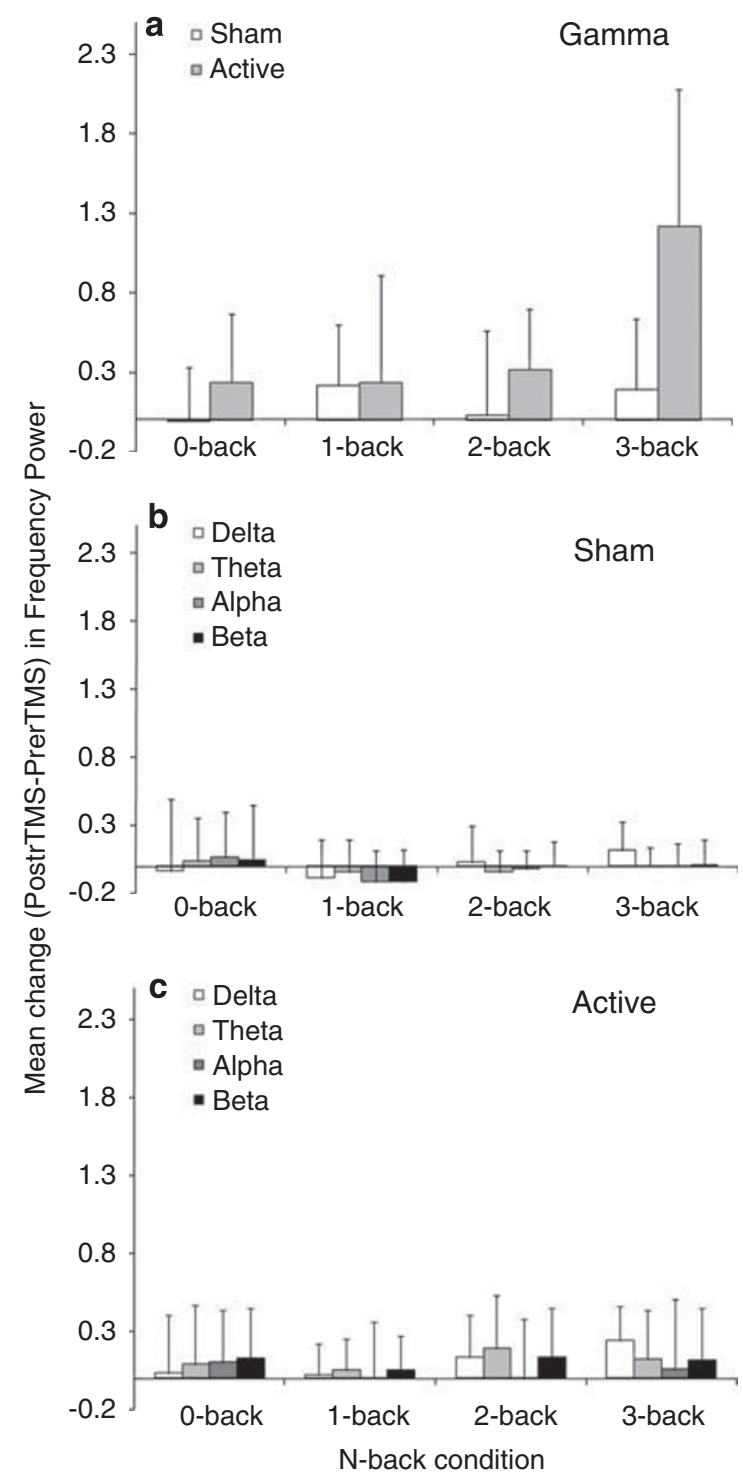

Figure 3 Mean log-transformed change in oscillatory power (post rTMS frequency power-pre rTMS frequency power) after rTMS elicited during the $N$-back task for (a) $\gamma$-power compared with $\delta, \theta, \alpha$, and $\beta$ activities after (b) sham and (c) active stimulation. Error bars represent $(+)$ standard deviation (SD).

$p<0.001$; Figure $4 \mathrm{~b}$ ) and the brain region $\times$ rTMS interaction was also significant $\left(F_{(1,19)}=11.445, p<0.005\right)$. Finally, paired $t$-tests revealed that this interaction was because of the difference between brain regions after active stimulation $\left(t_{(10)}=4.101, p<0.005\right.$; Figure $\left.4 \mathrm{~b}\right)$, whereas no difference between brain regions was found after sham stimulation $\left(t_{(9)}=0.619, p>0.05\right.$; Figure $4 \mathrm{~b}$; SPSS 15.0, SPSS Chicago, IL). These results further suggest that active rTMS resulted in a significant potentiation in $\gamma$-power isolated to the frontal brain regions during $N$-back conditions with the greatest WM load.

\section{EEG Spectral Analysis of other Frequency Bands}

To test whether our findings of enhanced mean $\gamma$-power after active rTMS elicited during the $N$-back task were selective to 
this frequency band, four separate MMRM analyses (active $v s$ sham; Figure 3b, c) as a between-subject factor and time (pre $v s$ post), and WM load (0- vs 1- vs 2- vs 3-back) as withinsubject factors were performed on the log-transformed mean evoked $\delta(1-3.5 \mathrm{~Hz}), \theta(4-8 \mathrm{~Hz}), \alpha(9-12 \mathrm{~Hz})$, and $\beta(14-$ $28 \mathrm{~Hz}$ ) power for TC and NTC responses. An effect of WM load was revealed in all frequency bands; however, there were no differences between active and sham stimulation under any WM load. Finally, a time $\times$ group interaction was also revealed in the $\theta$ - and $\beta$-frequency bands, but again with no difference between active and sham frequency power at either baseline (pre) or after rTMS (post). These subsequent
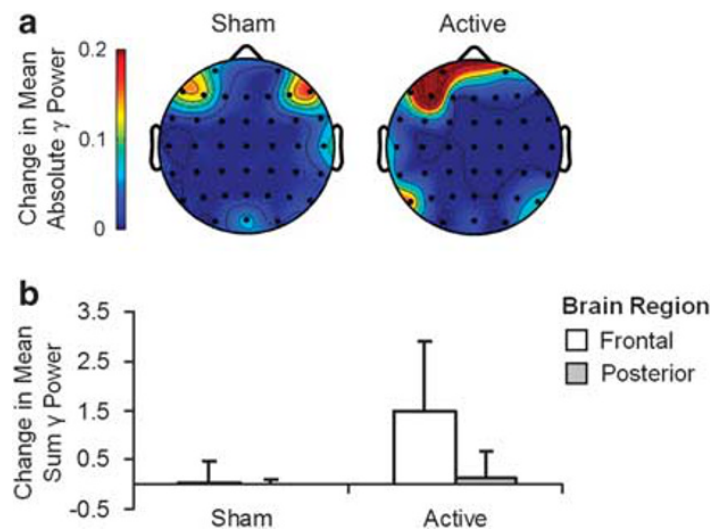

Figure 4 (a) Topographical illustration of mean absolute change (post rTMS $\gamma$-power-pre rTMS $\gamma$-power) in $\gamma$-power elicited during 3-back condition after sham and active rTMS. Maximal change in $\gamma$-power (represented by hot colors) was found after active stimulation in the frontal brain regions. (b) Change in the mean sum of $\gamma$-power in the frontal (FPZ $+\mathrm{FPI}+\mathrm{FP} 2+\mathrm{AF} 3+\mathrm{AF} 4)$ vs posterior $(\mathrm{OZ}+\mathrm{OI}+\mathrm{O} 2+\mathrm{PO} 3+\mathrm{PO} 4)$ electrodes in the 3 -back condition after sham and active stimulation. Error bars represent $(+)$ standard deviation (SD). spectral analyses thus show that active stimulation selectively enhanced the oscillatory activity in the $\gamma$-band only, whereas the activity in the other frequencies remained unchanged.

\section{$N$-Back WM Performance}

As expected, the MMRM analysis on WM performance revealed a significant effect of WM load $\left(F_{(2,40)}=78.63\right.$, $p<0.001)$ with performance decreasing with increasing cognitive demand. In particular, participants performed significantly better in the 1-back compared with the $2-(p=0.0005)$ and 3-back $(p<0.0001)$ conditions and better in the 2-back relative to the 3-back $(p<0.0001)$ condition, respectively (Table 2 ). However, the interaction between time and group was not significant, reflecting similar WM performance across groups (ie, active $v s$ sham) pre- and post rTMS $\left(F_{(1,20)}=0.31, p=0.5859\right)$. Finally, no relationship was found between mean WM performance and mean $\gamma$-power within any $N$-back condition determined through a Pearson's correlation coefficient.

As expected, we found an effect of WM load on reaction time $\left(F_{(3,60)}=126.64, p<0.0001\right.$; Table 3$)$ with reaction time increasing with increasing WM load. Specifically, pairwise comparisons revealed significantly lower RT in the 0-back relative to the $1-3$-back conditions ( $p<0.0001$ in each case), whereas reaction time was lower in the 1-back compared with the 2- $(p=0.0001)$ and 3-back condition $(p<0.0001)$, respectively.

\section{DISCUSSION}

We measured the effect of $20 \mathrm{~Hz}$ rTMS applied to the DLPFC on $\gamma$-oscillatory activity across WM load (ie, 0-, 1-, and 2back conditions). As predicted, $\gamma$-oscillatory activity generally increased with the increased WM load. Active rTMS

Table 2 Performance Across N-back Task Condition for Target and Non-Target Correct Responses Expressed as Percentage (\%) Before and After Sham or Active rTMS

\begin{tabular}{|c|c|c|c|c|c|c|}
\hline \multirow[t]{2}{*}{ Group } & \multicolumn{3}{|c|}{ Pre rTMS } & \multicolumn{3}{|c|}{ Post rTMS } \\
\hline & I-back & 2-back & 3-back & I-back & 2-back & 3-back \\
\hline Active $( \pm)$ ISD & $83.1( \pm) \mid 1.6$ & $74.1( \pm) 16.3$ & $66.8( \pm) \quad 11.5$ & $83.5( \pm) 7.8$ & $72.4( \pm) \quad 13.3$ & $64.1( \pm) 13.9$ \\
\hline
\end{tabular}

Data expressed as mean $( \pm)$ standard deviation $(\mathrm{SD})$.

Table 3 Reaction Time Across N-back Task Condition for Target and Non-Target Correct Responses Expressed in Milliseconds (ms) Before and After Sham or Active rTMS

\begin{tabular}{|c|c|c|c|c|c|c|c|c|}
\hline \multirow[t]{2}{*}{ Group } & \multicolumn{4}{|c|}{ Pre rTMS } & \multicolumn{4}{|c|}{ Post rTMS } \\
\hline & 0-back & I-back & 2-back & 3-back & 0-back & I-back & 2-back & 3-back \\
\hline Active $( \pm)$ ISD & $393.0( \pm) \quad 122.3$ & $793.6( \pm) \quad 155.1$ & $924.4( \pm) 257.4$ & $990.4( \pm) 317.1$ & $435.0( \pm) \quad 168.9$ & $756.7( \pm) 90.7$ & $862.0( \pm) 204.2$ & $956.4( \pm) 257.8$ \\
\hline
\end{tabular}

Data expressed as mean ( \pm ) standard deviation $(\mathrm{SD})$. 
significantly increased $\gamma$-oscillatory activity compared with baseline (pre) and sham stimulation. Moreover, active rTMS caused the greatest change in $\gamma$-oscillatory activity in the $\mathrm{N}$-back conditions with the greatest cognitive demand, an effect that was limited to the frontal brain regions. Finally, active rTMS had no effect on other frequency ranges (ie, $\delta$, $\theta, \alpha$, and $\beta$ ), suggesting a selective effect to oscillatory activity in the $\gamma$-frequency range. Collectively, these results suggest that active rTMS applied bilaterally to the DLPFC significantly increased frontal $\gamma$-oscillatory activity, which was most pronounced at $\mathrm{N}$-back conditions of greatest difficulty.

The finding that $\gamma$-oscillatory activity was most pronounced at $N$-back conditions of greatest difficulty is consistent with earlier studies examining the role of $\gamma$-oscillatory activity and WM (Basar-Eroglu et al, 2007; Howard et al, 2003; Meltzer et al, 2008). For example, Howard et al (2003) first showed a linear increase in $\gamma$-oscillatory power with WM load in epileptic patients performing the Sternberg WM task (Howard et al, 2003). Additional studies confirmed enhanced $\gamma$-oscillatory activity with WM load using both the Sternberg (Meltzer et al, 2008) and $N$-back (Basar-Eroglu et al, 2007) tasks. In these studies, however, WM loads comparable with the 3-back condition were not examined. In this regard, we observed significantly lower $\gamma$-oscillatory activity in the 3-back condition relative to the 2 -back condition at baseline in both groups $(p<0.0004)$. This finding is consistent with decreased BOLD activity elicited while healthy individuals performed the 3-back condition in fMRI studies. It has been suggested that these decreases in BOLD activity may be because WM resources are exceeded during the 3-back condition, resulting in poorer cognitive performance because of diminished attentional resources (Cowan, 2001; Kane et al, 2001; Wheeler and Treisman, 2002). Although speculative, the fact that active rTMS to the DLPFC resulted in an increase in $\gamma$-oscillatory activity, with the greatest effect in the 3-back condition (post; Figure 3a), suggests that rTMS, in part, may enhance attentional resources underlying such effects.

High-frequency rTMS has been shown to improve cognitive performance (Little et al, 2000; Sparing et al, 2001; Martis et al, 2003; O'Connor et al, 2005), however, the underlying mechanisms have not been investigated. We contend that a possible explanation is through its effects on GABAergic inhibitory neurotransmission critical in both the generation and synchronization of oscillatory activity (Whittington et al, 1995; Wang and Buzsaki, 1996; Bartos et al, 2007). GABAergic neurons in cortical networks interact with other neurons to affect spike timing and to coordinate rhythmic population activity (Mann and Paulsen, 2007). GABAergic neurons have also been implicated in the synchronization of pyramidal neurons in the DLPFC during WM (Lewis et al, 2005). For instance, Wilson et al (1994) showed that in monkeys, fast-spiking GABAergic neurons in the DLPFC remain active during the delay period of WM tasks (Wilson et al, 1994). In addition, during WM tasks, the activity of these GABAergic neurons contribute to the spatial tuning of the neuronal response, and are thus considered to be related to the WM task itself (Rao et al, 2000), whereas the injection of GABAergic antagonists in the DLPFC resulted in a disruption in WM performance (Sawaguchi et al, 1989). Taken together with the finding that $20 \mathrm{~Hz}$ rTMS potentiates GABAergic inhibitory neurotransmission indexed through CSP (Daskalakis et al, 2006), we contend that high-frequency rTMS exerts its effects on $\gamma$-oscillatory activity through GABA receptor-mediated inhibitory neurotransmission to ultimately affect WM performance. Future studies combining paired-pulse TMS with EEG recording to index GABA receptor-mediated neurotransmission in the DLPFC (Daskalakis et al, 2008) will be needed, however, to determine whether $\gamma$-oscillatory activity is indeed potentiated directly through enhanced GABA.

Earlier studies have also reported modulations in other frequency ranges during cognitive tasks. Although changes in oscillatory activity in the $\gamma$-frequency band are most consistently observed, increases in the $\theta-, \alpha$-, and $\beta$-frequency bands have also been associated with cognitive tasks (Gevins et al, 1997; Klimesch et al, 2001; Klimesch et al, 1997; Klimesch, 1999; Sarnthein et al, 1998; TallonBaudry et al, 1999; von Stein et al, 1999; Tesche and Karhu, 2000; Raghavachari et al, 2001; Jensen et al, 2002; Schack et al, 2002; Howard et al, 2003; Rizzuto et al, 2003; Gruber and Muller, 2005; Kaiser and Lutzenberger, 2005). In line with these studies, we found an increase in $\delta$-, $\theta-, \alpha$-, and $\beta$ - activity with WM load. However, after rTMS applied to the DLPFC, modulations were only found within the $\gamma$-frequency range, whereas $\delta$-, $\theta-, \alpha$-, and $\beta$-activities remained unchanged. These findings indicate that $\delta-, \theta-$, $\alpha-, \beta$-, and $\gamma$-activities are modulated by cognitive demand, yet only $\gamma$-activity was enhanced through rTMS on the DLPFC - a brain region that is closely associated with attention and WM performance.

To our knowledge, this is the first demonstration of enhanced $\gamma$-oscillatory activity elicited during the $N$-back task after a single session of rTMS on the DLPFC. Although $\gamma$-oscillatory activity was potentiated after rTMS, this effect was not related to WM performance. It may be possible that rTMS-induced cognitive changes are either delayed or optimal at some later time point and/or that repeated sessions (ie, days to weeks) of rTMS are needed for such effects to be fully realized. In this experiment, individuals were tested in the $\mathrm{N}$-back task for a second time within 20 min after rTMS administration, which may have adequately captured the neurophysiological but not the anticipated cognitive changes. This contention is consistent with literature from rTMS treatment studies. For example, it has been suggested that repeated sessions of rTMS may be necessary to produce changes in gene expression and synapse formation that accompany changes in short-term plasticity and cognition (Khedr et al, 2006). Further studies, therefore, are needed to determine whether the anticipated changes to WM performance, which may follow enhanced $\gamma$-oscillatory activity, are either delayed or require multiple rTMS sessions to be fully realized.

Oscillations in the $\gamma$-frequency range are typically estimated using two sub-classifications that differ in their phase relationship with respect to the stimulus onset. Evoked $\gamma$-oscillatory activity is phase locked to the stimulus and occurs $\sim 200 \mathrm{~ms}$ relative to stimulus onset (TallonBaudry et al, 1999). As evoked $\gamma$-oscillatory activity is phase locked to the stimulus, these responses can be extracted in a time domain and averaged across trials (Pantev, 1995). 
In contrast, induced $\gamma$-oscillatory activity is not phase locked to the stimulus, but rather jitters in latency from trial to trial and, thus, cannot be averaged across trials. Induced $\gamma$-oscillatory activity is measured by applying the timefrequency decomposition to each trial and the ensuing power is averaged across trials. The power of evoked and background components are then subtracted from the total power to provide an estimate of induced $\gamma$-oscillatory activity (David et al, 2006). Although there are numerous reports that support a relationship between induced $\gamma$-oscillatory activity and cognitive functions (Cho et al, 2006; Gray et al, 1989; Fries et al, 2001; Pesaran et al, 2002), a recent study showed that measures of induced $\gamma$-activity may not only reflect synchronous neuronal activity, but also the activation of miniature saccadic eye movements (YuvalGreenberg et al, 2008). In their study, Yuval-Greenberg et al (2008) recorded eye movements simultaneously with EEG and found that induced $\gamma$-activity was time locked to the onset and the rate of involuntary miniature saccades and thus reflects saccadic spike potentials rather than neuronal oscillations. These authors contend that measures of evoked $\gamma$-activity may better mitigate such effects from miniature saccadic activity on this neurophysiological phenomenon (Yuval-Greenberg et al, 2008). Considering that induced $\gamma$-activity may reflect involuntary eye movements with emerging evidence supporting the association of evoked $\gamma$-activity with WM (Howard et al, 2003; Meltzer et al, 2008; Basar-Eroglu et al, 2007), we selected to measure the effect of rTMS on evoked $\gamma$-activity.

The results of this study are limited by the relatively small sample size, which may be attributed to the lack of a relationship found between increased $\gamma$-oscillatory activity and WM performance. Replication studies assessing the effect of rTMS on WM may consider using a larger sample size to further examine this relationship. However, the fact that rTMS enhanced $\gamma$-oscillatory activity compared with sham, which was not observed within the other frequency ranges, suggests that the observed potentiation of $\gamma$-oscillatory activity was not related to the small sample size. Nevertheless, such findings should be replicated in a larger sample to minimize type II error and stabilize statistical parameter estimates (Norman and Streiner, 2000).

A second limitation to this study is the use of the $N$-back task to evaluate $\gamma$-oscillatory activity underlying WM. Earlier studies that have examined the convergent validity between the $N$-back task and other WM measures, such as the operation span task (OSPAN), show correlations with lower WM loads (Shelton et al, 2007) and varied results with the 3-back condition (Shelton et al, 2007; Kane et al, 2007). Such studies suggest that at higher WM loads, attentional components and/or short-term memory processes may be measured rather than WM capacity exclusively. Our finding of increased $\gamma$-oscillatory activity after rTMS on the DLPFC may, therefore, reflect enhancements in attention and/or short-term memory processes rather than WM capacity exclusively. A third limitation to this study is the time delay between rTMS administration and the measurement of the $\mathrm{N}$-back task - that is, participants were tested $20 \mathrm{~min}$ after rTMS, though not immediately afterward to allow for the placement of the EEG cap. It is possible, therefore, that both neurophysiological and perhaps behavioral changes may have been more optimally measured immediately after
rTMS administration but missed because of this technical limitation. A final important limitation to this study is that we tested individuals at only a single time point (ie, within $20 \mathrm{~min}$ ) after a single session of rTMS and, therefore, we were unable to examine the time course of these neurophysiological changes and indicate whether such changes persist, increase or decay over time, and/or whether such changes are even further enhanced and possibly translated into more persistent neurophysiological and possibly behavioral changes through repeated rTMS sessions. However, despite these limitations, our findings provide a neurophysiological framework through which to evaluate rTMS as a therapeutic tool in patient populations (eg, schizophrenia), in which WM deficits form part of the symptom profile.

In summary, we show that high-frequency rTMS on the DLPFC significantly enhanced frontal $\gamma$-oscillatory elicited in $N$-back conditions with the greatest task difficulty. Furthermore, rTMS administered to the DLPFC selectively increased oscillatory activity in the $\gamma$-frequency range, whereas other frequency ranges (ie, $\delta, \theta, \alpha$, or $\beta$ ) remained unchanged. The specificity of high-frequency rTMS on $\gamma$-oscillatory activity found in this study may, therefore, provide important insights into the pathophysiology of brain disorders that present cognitive deficits as part of their symptom profiles.

\section{DISCLOSURE/CONFLICT OF INTEREST}

This work was funded, in part, by the Canadian Institutes of Health Research (CIHR) (Grant no. MOP 62917 to RC), CIHR Clinician Scientist Award (ZJD), by an operating and studentship award from the Ontario Mental Health Foundation (ZJD and $\mathrm{MB}$, respectively), by a National Health and Medical Research Council (NHMRC) Practitioner Fellowship (PBF), and by Constance and Stephen Lieber through a National Alliance for Research on Schizophrenia and Depression (NARSAD) Lieber Young Investigator award (ZJD, PBF). ZJD and PBF have both received external funding through Neuronetics and ZJD has also received external funding through Aspect Medical and travel support through Pfizer.

\section{ACKNOWLEDGEMENTS}

We gratefully acknowledge the assistance of all persons and volunteers whose participation was essential in the successful completion of the study.

\section{REFERENCES}

Baddeley A (1986). Working Memory. Claredon Press: Oxford. Baddeley A (1992). Working memory. Science 255: 556-559.

Baddeley A (2000). The episodic buffer: a new component of working memory? Trends Cogn Sci 4: 417-423.

Bartos M, Vida I, Jonas P (2007). Synaptic mechanisms of synchronized gamma oscillations in inhibitory interneuron networks. Nat Rev Neurosci 8: 45-56.

Basar-Eroglu C, Brand A, Hildebrandt H, Karolina Kedzior K, Mathes B, Schmiedt C (2007). Working memory related 
gamma oscillations in schizophrenia patients. Int J Psychophysiol 64: 39-45.

Bender R, Schultz B, Schultz A, Pichlmayr I (1992). Testing the gaussianity of the human EEG during anesthesia. Methods Inf Med 31: 56-59.

Cannon TD, Glahn DC, Kim J, Van Erp TG, Karlsgodt K, Cohen MS et al (2005). Dorsolateral prefrontal cortex activity during maintenance and manipulation of information in working memory in patients with schizophrenia. Arch Gen Psychiatry 62: 1071-1080.

Chen R, Gerloff C, Classen J, Wassermann EM, Hallett M, Cohen LG (1997). Safety of different inter-train intervals for repetitive transcranial magnetic stimulation and recommendations for safe ranges of stimulation parameters. Electroencephalogr Clin Neurophysiol 105: 415-421.

Cho RY, Konecky RO, Carter CS (2006). Impairments in frontal cortical gamma synchrony and cognitive control in schizophrenia. Proc Natl Acad Sci USA 103: 19878-19883.

Cowan N (2001). The majical number 4 in short-term memory: a reconsideration of mental storage capacity. Behav Brain Sci 24: 87-114.

Daskalakis ZJ, Farzan F, Barr MS, Maller JJ, Chen R, Fitzgerald PB (2008). Long-interval cortical inhibition from the dorsolateral prefrontal cortex: a TMS-EEG study. Neuropsychopharmacology 33: $2860-2869$.

Daskalakis ZJ, Moller B, Christensen BK, Fitzgerald PB, Gunraj C, Chen $R$ (2006). The effects of repetitive transcranial magnetic stimulation on cortical inhibition in healthy human subjects. Exp Brain Res 174: 403-412.

David O, Kilner JM, Friston KJ (2006). Mechanisms of evoked and induced responses in MEG/EEG. Neuroimage 31: 1580-1591.

Delorme A, Makeig S (2004). EEGLAB: an open source toolbox for analysis of single-trial EEG dynamics including independent component analysis. J Neurosci Methods 134: 9-21.

Fries P, Reynolds JH, Rorie AE, Desimone R (2001). Modulation of oscillatory neuronal synchronization by selective visual attention. Science 291: 1560-1563.

Gevins A, Smith ME, McEvoy L, Yu D (1997). High-resolution EEG mapping of cortical activation related to working memory: effects of task difficulty, type of processing, and practice. Cereb Cortex 7: 374-385.

Gray CM, Konig P, Engel AK, Singer W (1989). Oscillatory responses in cat visual cortex exhibit inter-columnar synchronization which reflects global stimulus properties. Nature 338: 334-337.

Gruber T, Muller MM (2005). Oscillatory brain activity dissociates between associative stimulus content in a repetition priming task in the human EEG. Cereb Cortex 15: 109-116.

Howard MW, Rizzuto DS, Caplan JB, Madsen JR, Lisman J, Aschenbrenner-Scheibe $\mathrm{R}$ et al (2003). Gamma oscillations correlate with working memory load in humans. Cereb Cortex 13: $1369-1374$.

Jensen O, Gelfand J, Kounios J, Lisman JE (2002). Oscillations in the alpha band $(9-12 \mathrm{~Hz})$ increase with memory load during retention in a short-term memory task. Cereb Cortex 12: $877-882$.

Kaiser J, Lutzenberger W (2005). Human gamma-band activity: a window to cognitive processing. Neuroreport 16: 207-211.

Kane MJ, Bleckley MK, Conway AR, Engle RW (2001). A controlled-attention view of working-memory capacity. $j$ Exp Psychol Gen 130: 169-183.

Kane MJ, Conway AR, Miura TK, Colflesh GJ (2007). Working memory, attention control, and the N-back task: a question of construct validity. J Exp Psychol Learn Mem Cogn 33: 615-622.

Khedr EM, Rothwell JC, Shawky OA, Ahmed MA, Hamdy A (2006). Effect of daily repetitive transcranial magnetic stimulation on motor performance in Parkinson's disease. Mov Disord 21: 2201-2205.

Klimesch W (1999). EEG alpha and theta oscillations reflect cognitive and memory performance: a review and analysis. Brain Res Brain Res Rev 29: 169-195.

Klimesch W, Doppelmayr M, Schimke H, Ripper B (1997). Theta synchronization and alpha desynchronization in a memory task. Psychophysiology 34: 169-176.

Klimesch W, Doppelmayr M, Stadler W, Pollhuber D, Sauseng P, Rohm D (2001). Episodic retrieval is reflected by a process specific increase in human electroencephalographic theta activity. Neurosci Lett 302: 49-52.

Lewis DA, Hashimoto T, Volk DW (2005). Cortical inhibitory neurons and schizophrenia. Nat Rev Neurosci 6: 312-324.

Little JT, Kimbrell TA, Wassermann EM, Grafman J, Figueras S, Dunn RT et al (2000). Cognitive effects of 1- and 20-hertz repetitive transcranial magnetic stimulation in depression: preliminary report. Neuropsychiatry Neuropsychol Behav Neurol 13: 119-124.

Lutzenberger W, Pulvermuller F, Elbert T, Birbaumer N (1995). Visual stimulation alters local $40-\mathrm{Hz}$ responses in humans: an EEG-study. Neurosci Lett 183: 39-42.

Mann EO, Paulsen O (2007). Role of GABAergic inhibition in hippocampal network oscillations. Trends Neurosci 30: 343-349.

Martis B, Alam D, Dowd SM, Hill SK, Sharma RP, Rosen C et al (2003). Neurocognitive effects of repetitive transcranial magnetic stimulation in severe major depression. Clin Neurophysiol 114: 1125-1132.

Meltzer JA, Zaveri HP, Goncharova II, Distasio MM, Papademetris $\mathrm{X}$, Spencer SS et al (2008). Effects of working memory load on oscillatory power in human intracranial EEG. Cereb Cortex 18: 1843-1855.

Mendrek A, Kiehl KA, Smith AM, Irwin D, Forster BB, Liddle PF (2005). Dysfunction of a distributed neural circuitry in schizophrenia patients during a working-memory performance. Psychol Med 35: 187-196.

Muller MM, Bosch J, Elbert T, Kreiter A, Sosa MV, Sosa PV et al (1996). Visually induced gamma-band responses in human electroencephalographic activity - a link to animal studies. Exp Brain Res 112: 96-102.

Norman GR, Streiner DL (2000). Biostatistics - The Bare Essentials, 2nd edn B.C. Decker Inc: Hamilton.

O'Connor MG, Jerskey BA, Robertson EM, Brenninkmeyer C, Ozdemir E, Leone AP (2005). The effects of repetitive transcranial magnetic stimulation (rTMS) on procedural memory and dysphoric mood in patients with major depressive disorder. Cogn Behav Neurol 18: 223-227.

Oldfield RC (1971). The assessment and analysis of handedness: the Edinburgh inventory. Neuropsychologia 9: 97-113.

Owen AM (1997). The functional organization of working memory processes within human lateral frontal cortex: the contribution of functional neuroimaging. Eur J Neurosci 9: 1329-1339.

Pantev C (1995). Evoked and induced gamma-band activity of the human cortex. Brain Topogr 7: 321-330.

Pesaran B, Pezaris JS, Sahani M, Mitra PP, Andersen RA (2002). Temporal structure in neuronal activity during working memory in macaque parietal cortex. Nat Neurosci 5: 805-811.

Petrides M (2000). The role of the mid-dorsolateral prefrontal cortex in working memory. Exp Brain Res 133: 44-54.

Raghavachari S, Kahana MJ, Rizzuto DS, Caplan JB, Kirschen MP, Bourgeois B et al (2001). Gating of human theta oscillations by a working memory task. J Neurosci 21: 3175-3183.

Rao SG, Williams GV, Goldman-Rakic PS (2000). Destruction and creation of spatial tuning by disinhibition: $\operatorname{GABA}(\mathrm{A})$ blockade of prefrontal cortical neurons engaged by working memory. J Neurosci 20: 485-494.

Rizzuto DS, Madsen JR, Bromfield EB, Schulze-Bonhage A, Seelig $\mathrm{D}$, Aschenbrenner-Scheibe $\mathrm{R}$ et al (2003). Reset of human 
neocortical oscillations during a working memory task. Proc Natl Acad Sci USA 100: 7931-7936.

Sarnthein J, Petsche H, Rappelsberger P, Shaw GL, von Stein A (1998). Synchronization between prefrontal and posterior association cortex during human working memory. Proc Natl Acad Sci USA 95: 7092-7096.

Sawaguchi T, Matsumura M, Kubota K (1989). Delayed response deficits produced by local injection of bicuculline into the dorsolateral prefrontal cortex in Japanese macaque monkeys. Exp Brain Res 75: 457-469.

Schack B, Vath N, Petsche H, Geissler HG, Moller E (2002). Phasecoupling of theta-gamma EEG rhythms during short-term memory processing. Int J Psychophysiol 44: 143-163.

Shelton JT, Metzger RL, Elliott EM (2007). A group-administered lag task as a measure of working memory. Behav Res Methods 39: 482-493.

Sparing R, Mottaghy FM, Hungs M, Brugmann M, Foltys H, Huber $\mathrm{W}$ et al (2001). Repetitive transcranial magnetic stimulation effects on language function depend on the stimulation parameters. J Clin Neurophysiol 18: 326-330.

Tallon-Baudry C, Kreiter A, Bertrand O (1999). Sustained and transient oscillatory responses in the gamma and beta bands in a visual short-term memory task in humans. Vis Neurosci 16: 449-459.

Tan HY, Choo WC, Fones CS, Chee MW (2005). fMRI study of maintenance and manipulation processes within working memory in first-episode schizophrenia. Am J Psychiatry 162: $1849-1858$.
Tesche CD, Karhu J (2000). Theta oscillations index human hippocampal activation during a working memory task. Proc Natl Acad Sci USA 97: 919-924.

Traub RD, Michelson-Law H, Bibbig AE, Buhl EH, Whittington MA (2004). Gap junctions, fast oscillations and the initiation of seizures. Adv Exp Med Biol 548: 110-122.

von Stein A, Rappelsberger P, Sarnthein J, Petsche H (1999). Synchronization between temporal and parietal cortex during multimodal object processing in man. Cereb Cortex 9: 137-150.

Wang XJ, Buzsaki G (1996). Gamma oscillation by synaptic inhibition in a hippocampal interneuronal network model. J Neurosci 16: 6402-6413.

Wheeler ME, Treisman AM (2002). Binding in short-term memory. $J$ Exp Psychol Gen 131: 48-64.

Whittington MA, Traub RD, Jefferys JG (1995). Synchronized oscillations in interneuron networks driven by metabotropic glutamate receptor activation. Nature 373: 612-615.

Wilson FA, O'Scalaidhe SP, Goldman-Rakic PS (1994). Functional synergism between putative gamma-aminobutyrate-containing neurons and pyramidal neurons in prefrontal cortex. Proc Natl Acad Sci USA 91: 4009-4013.

Yuval-Greenberg S, Tomer O, Keren AS, Nelken I, Deouell LY (2008). Transient induced gamma-band response in EEG as a manifestation of miniature saccades. Neuron 58: 429-441.

Ziemann U, Lonnecker S, Steinhoff BJ, Paulus W (1996). The effect of lorazepam on the motor cortical excitability in man. Exp Brain Res 109: 127-135. 